\title{
La gobernabilidad metropolitana de Santiago: la dispar relación de poder de los municipios
}

\author{
ARTURO ORELLANA** \\ ** Profesor investigador Instituto de Estudios Urbanos y Territoriales, Pontificia Universidad Católica de Chile.
}

\begin{abstract}
In the absence of metropolitan level government, large cities retain the problems associated with multilevel management whereby the activities of central government, regional government and numerous municipal governments overlap, generating in turn a complex scenario for governance in favour of balanced urban and regional development. From this perspective, the significant disparity in socio-spatial configuration within the Santiago Metropolitan Area reflects the structure of power within this metropolitan space, whereby municipalities in the eastern cone are better able to shape public and private actions in support of the public interests of their resident populations.
\end{abstract}

KEYWORDS: municipalities, metropolitan government, power, local government, governability.

RESUMEN En ausencia de un gobierno metropolitano, las grandes ciudades que sostienen la problemática de una gestión multinivel, es decir, donde se sobrepone la acción del gobierno central, regional y de un conjunto significativo de municipios, generan un escenario complejo para la gobernabilidad en pro de un desarrollo urbano y territorial equilibrado. Desde esta perspectiva, la gran disparidad en la configuración socioterritorial que muestra el Área Metropolitana de Santiago constituye un reflejo de la estructura de poder sobre el espacio metropolitano, donde las comunas del cono oriente muestran un mejor posicionamiento para orientar las acciones públicas y privadas a favor del interés público de su población residente.

PALABRAS CLAVES: municipios, gobierno metropolitano, poder, gestión local, gobernabilidad.

Este artículo se enmarca dentro de los avances de la investigación del proyecto FONDECYT 11085032 Recibido el 23 de julio de 2008, aprobado el 23 de enero de 2009.

Correspondencia: El Comendador 1916, Casilla 16002, Correo 9, Santiago de Chile. E-mail: amorella@uc.cl. 


\section{Antecedentes}

El presente artículo constituye un avance en la investigación que lleva actualmente el autor para el caso del Área Metropolitana de Santiago de Chile. Se inserta dentro de una línea de trabajo que se inició con una investigación realizada en años recientes para el caso del Área Metropolitana de Barcelona (AMB), materializada en la tesis doctoral "La incidencia de la gestión municipal en la gobernabilidad metropolitana del Área Metropolitana de Barcelona”.

El propósito de la investigación realizada en su oportunidad, fue desarrollar y aplicar una metodología que permitiera establecer en áreas metropolitanas cómo la orientación de la gestión municipal puede influir en la gobernabilidad metropolitana, particularmente de aquellos municipios donde la población residente tiene un perfil socioeconómico superior. En este intento, la propuesta metodológica combinó en el análisis de forma pionera fuentes cualitativas y cuantitativas de carácter secundario. En una primera fase de investigación, se buscó probar que en las áreas metropolitanas existe una disparidad significativa de perfiles socioterritoriales entre municipios. En base a la aplicación de esta metodología, se comprobó que para el caso del AMB coexisten municipios cuya población presenta, por un lado, un alto grado de educación y pertenece a la categoría sociocupacional de Dirigentes (directivos públicos y privados), con escasa o casi nula población en situación de pobreza, donde el municipio detenta un alto presupuesto per cápita, el cual no se explica por los tamaños de población a escala local. Y, por otro lado, municipios que muestran un perfil socioterritorial totalmente opuesto.

A partir de dicha constatación, se exploró en la relación que existía entre los perfiles socioterritoriales de los municipios y el tipo de demandas sobre la gestión municipal, partiendo de una hipótesis preliminar consistente en probar si es que el perfil socioterritorial de la población residente determina en forma importante las actuaciones de la gestión municipal. Esta hipótesis, que en apariencia se podría haber considerado de total obviedad, debía ser probada, lo que llevó a contrastarla con la información contenida en las actas municipales para un período de cinco años, para lo cual se compararon las actas de los cinco municipios del extremo superior con los cinco del extremo inferior del ranking. A partir de esta constatación, fue posible plantearse una segunda hipótesis de trabajo, consistente en la sospecha que la gestión municipal de los municipios de perfil socioterritorial superior se enfocaria preferentemente hacia actuaciones que tiene un impacto más metropolitano que local. Esta hipótesis, para el caso del AMB, fue validada, a partir también de comprobar que las noticias relativas a la gestión municipal que alcanzaban presencia en los medios de prensa escrito, para el mismo período que se revisaron las actas municipales, se comportaba de la misma forma. Y, así también, había total correspondencia entre los objetivos de trabajo de las organizaciones no gubernamentales con personalidad jurídica con domicilio en una comuna, y el perfil de sus residentes.

Todo lo anterior sintetiza el proceso metodológico con el que se está trabajando el caso del Área Metropolitana de Santiago (AMS), siendo este artículo un primer esfuerzo de reflexión

La Comisión Doctoral fue integrada por los doctores Horacio Capel (Presidente), Albert Serratosa, Jaume Font, Inmaculada Caravaca y Ana Alabart, en la Universitat de Barcelona, abril 2006. 
y análisis sobre la dispar relación de poder de los municipios en esta metrópolis, que se puede deducir de los marcados diferenciales que surgen del ranking de perfiles socioterritoriales construidos sobre la base del método aplicado por Sánchez (1997) para el caso de los municipios de Cataluña. Sin embargo, previo a mostrar esos resultados, se entrega un acabado marco teórico que sustenta la relación entre lo metropolitano, la gestión de los municipios y la gobernabilidad, donde se encuentran fundamentos de peso para relacionar el poder de los municipios con la gobernabilidad metropolitana, aun sin haber terminado la investigación en curso para el AMS.

\section{De lo metropolitano y lo local}

El territorio metropolitano se articula en una superposición de varios niveles político-administrativos, siendo en gran parte de los casos común que este espacio lo configuren un número significativo de municipios, y que esté además bajo un gobierno de escala territorial superior: regional, provincial y departamental, siendo una excepción la existencia de alguna Alcaldía Mayor o Gobierno Metropolitano. La resistencia a la institucionalización metropolitana, según Borja (2008) se manifiesta por dos frentes. Por un lado, la cesión de competencias desde el nivel superior y desde el nivel inferior de gobierno y, por otro lado, debido al rechazo a nuevos gobiernos por ser estructuras demasiado alejadas de la ciudadanía, lo que significa que ambas razones lleven a que en las áreas metropolitanas se instale un escenario de competencia no declarada entre municipios en la búsqueda de dar respuestas a las demandas de sus residentes.

Lo anterior obliga a una mejor comprensión de este fenómeno metropolitano, que deje atrás lo que entendíamos por ciudad: “aquella composición espacial definida por alta densidad poblacional y el asentamiento de un amplio conjunto de construcciones estables, una colonia humana densa y heterogénea conformada esencialmente por extraños entre sî" (Delgado, 1999, p. 23). Por el contrario, la metropolización trae consigo la multiplicación de espacios urbanizados, donde, como señala el mismo autor, "los vínculos son preferentemente laxos no forzosos, los intercambios aparecen en gran medida no programados, los encuentros más estratégicos pueden ser fortuitos, domina la incertidumbre sobre interacciones inminente, las informaciones más determinantes pueden ser obtenidas por casualidad y el grueso de las relaciones sociales se produce entre conocidos 'de vista'" (pp. 23-24).

En sintonía con lo anterior, se expresa en muchos casos lo que Soja (2000) en su libro Postmetropolis invoca con su definición de fractal city, aludiendo a un proceso de metro-polarización donde se recrudecen desigualdades en la ciudad a partir del fenómeno de crecimiento suburbano. Se trata de una geometría fracturada que incrementa las distancias territoriales y sociales, lo que genera un nuevo orden socio-urbano sobre la base de nuevos patrones de estratificación social, posible de advertir en una serie expresiones, como el crecimiento de la pobreza, la segregación espacial, el empobrecimiento de la clase media y la explosión social de los suburbios. Se instala un clima urbano amparado en el desconocimiento del vecino que alimenta una percepción de la ciudad del miedo y la concepción del ciudadano como usuario. Esto, en el marco de una globalización que se impone a escala planetaria, caracterizada por un proceso que genera espacios contradictorios, marcados por los conflictos, la diferenciación interna y los continuos cruces de límites, donde las metrópolis conectadas en la red de 
ciudades globales atraen el poder corporativo global y son a su vez uno de los sitios clave para su valorización (Sassen, 2003).

Sin embargo, se da la paradoja que en estas metrópolis donde se engendran las desigualdades, también son el lugar donde la población más desfavorecida por el sistema encuentra sus oportunidades de desarrollo. Es en estos espacios donde se expresan nuevas formas de gobernanza, dado que es el lugar donde están dadas las condiciones y la oportunidad de realizar sus reclamos sobre la ciudad, por ende, donde se concentra la mayor expresión del poder político y económico de la sociedad. El contexto contradictorio y dinámico de globalización como agente inductor del proceso de metropolización, genera múltiples cambios económicos, políticos, sociales, culturales y espaciales, que van configurando formas de organización socioespacial y territorial, que tensionan la necesidad de convivencia social (exclusión, segregación, fragmentación) como la distribución igualitaria de los recursos en el espacio territorial de las grandes urbes (Capel, 2003).

El espacio metropolitano, a su vez, se configura como el lugar con mayores ventajas para invertir, producir, recrearse y residir. El interés público se ve amenazado por el papel gravitante que ejercen sobre la gobernabilidad de la ciudad las clases directivas, aquellos que pueden elegir arbitrariamente y con total libertad dónde residir e incluso pagar impuestos, formas manifiestas del interés privado que emerge alterando la morfología de las nuevas urbes y también civitas. De alguna forma, como señala Molotch (1976), las ciudades son "máquinas de crecimiento" resultado en gran medida de políticas locales, fruto de batallas entre distintas fracciones sociales, y que crece según la disposición de las construcciones realizadas por distintos grupos bajo una lógica de mercado, avalados por los gobiernos regionales y/o urbanos.

Bajo la división político-administrativa entre los municipios que lo conforman, emerge una nueva ciudad extensiva articulada bajo relaciones dominantes en forma de red en la que los ciudadanos y ciudadanas deberán luchar para conseguir el equilibrio entre la calidad de los espacios vivenciales y la funcionalidad de sus actuaciones puntuales sobre el territorio amplio, sea para desplazarse a trabajar, a comprar, a estudiar, a divertirse, o para resolver sus problemas de salud (Sánchez, 1991). En una sociedad con individuos que no disfrutan de las mismas calidad de servicios en su ámbito local, hace que los distintos y diversos intereses de cada uno entren en conflicto y escalen hacia al ámbito metropolitano.

El grado de poder y de control que puede ejercer un municipio sobre su territorio en el contexto metropolitano, se establece en gran parte por la capacidad de gestión sobre su mismo territorio (Orellana, 2005). Sin embargo, dicha capacidad sobre una parte entra en conflicto con aquella gobernabilidad que se establece o se quiere establecer sobre el territorio metropolitano como un todo, una gestión de carácter institucional que abre una situación donde se traslapan competencias e intereses entre las actuaciones que derivan de la gestión municipal y aquellas políticas de alcance metropolitano que intentan imponerse. Desde esta perspectiva, se está en presencia de respuestas centralizadas del poder para gobernar un proceso de metropolización que, aunque parezca inadmisible, suelen exportarse de una realidad a otra sin establecer juicios sobre la naturaleza estructural y funcional del territorio. 
En resumen, las metrópolis son aquellos territorios de estructura reticular donde se desarrollan las actividades más dinámicas e innovadoras, pero también donde subsisten los mayores problemas de marginalidad, donde existe riqueza y bienestar, pero también donde es posible encontrar las mayores desigualdades y desequilibrios (Borja y Castells, 1998). Son los centros neurálgicos de explotación, donde las regiones e incluso los Estados se juegan sus posibilidades de inserción en la nueva economía global. Por tal motivo, suponen un entorno de conflicto social y espacial cuya dialéctica puede resolver el utopismo espacio-temporal (Harvey, 2000), donde subyacen y se sobreponen los intereses que todos aquellos referentes sociales que se disputan el devenir del territorio metropolitano.

\section{El Área Metropolitana de Santiago²}

El Área Metropolitana de Santiago (AMS) es el centro neurálgico de la Región Metropolitana (RM), una región que ocupa el $2 \%$ del territorio nacional, donde reside una población de 6,1 millones de habitantes, lo que representa el 40,1\% del total de la población que vive en el país y donde prácticamente el 95\% es población urbana. El Producto Interno Bruto (PIB) de la RM representa el 48\% del país, lo que hace que la dinámica económica de esta región supere largamente a la del resto del país. Así, el AMS no sólo es el motor de la economía nacional, sino la principal fuente de capital social y político del país.

En términos político-administrativos el AMS agrupa 34 comunas de las 52 comunas de la Región Metropolitana; 32 pertenecientes a la Provincia de Santiago, más las comunas de Puente Alto y San Bernardo, cuyos municipios son capitales de las provincias Cordillera y Maipo, respectivamente. En esta área metropolitana residen aproximadamente 5,5 millones de habitantes, más del $90 \%$ de la población total de la región.

Resultado de este proceso, a principios de los años noventa se fue afianzando progresivamente el patrón de segregación socioespacial y de espacialización territorial en el AMS que históricamente ha predominado en el desarrollo urbano de Santiago. Si bien este patrón se reconoce que es intrínseco a gran parte de las experiencias de metropolización a escala global -como lo advierte un reciente trabajo de Rojas, Cuadrado-Roura y Fernández Güell (2005)-, lo cierto es que para el caso específico del AMS no se condice con el nivel de desarrollo alcanzado por el país, que le permite liderar a nivel latinoamericano la gran mayoría de los rankings internacionales sobre esta materia.

Junto a ello, se debe considerar, tal como lo afirma Smith (2002), que para el caso del AMS hoy en día el Estado, en toda su expresión de escalas político-administrativas, no es el único órgano de decisión, pues ha ido cediendo parte de su "poder" y delegando cada vez más en otras instituciones (privadas y de la sociedad civil) responsabilidades que antes le eran

\footnotetext{
2 La descripción del Área Metropolitana de Santiago ha sido hecha de acuerdo al trabajo publicado recientemente por el autor en la Revista Ánfora, Universidad Autónoma de Manizales, 2007, ver bibliografía. De todas formas, los datos entregados pueden ser cotejados en el portal del Instituto Nacional de Estadísticas de Chile, www.ine. cl , y en la página del Sistema Nacional de Información Municipal (SINIM) de la Subsecretaría de Desarrollo Regional (SUBDERE), www.subdere.cl
} 
exclusivas. Por lo tanto, ahora existen diversos "lugares de decisión” y la forma de gobierno pasa a tener múltiples niveles.

\section{El papel de la gestión municipal}

En el libro El buen gobierno de la ciudad publicado por Joseph Centelles i Portella (2006), se consigna que no más del $8 \%$ de la economía de la ciudad sería generado a partir de las actuaciones de la administración municipal. Para este autor, la tarea de gobernar las ciudades consiste en tener la capacidad operativa de influenciar e intervenir de forma significativa en las redes de intereses que se conforman en la actuación de los diferentes agentes urbanos, ya sea en conflicto o en colaboración. Lo anterior, se logra por la vía de la provisión de los servicios públicos locales y por la regulación de actividades en el espacio urbano.

Es precisamente en la capacidad de actuar en esos dos ámbitos donde se hacen presentes las mayores disparidades entre los municipios. Una aproximación empírica se puede evidenciar de acuerdo a los resultados obtenidos en Orellana $(2006)^{3}$ para el caso del Área Metropolitana de Barcelona (AMB), donde se demuestra que un mayor perfil socioterritorial ${ }^{4}$ tiende a orientar su provisión de servicios públicos más hacia aspectos relativos al mejoramiento del entorno que a la entrega directa de subsidios para atender demandas sociales, así como a generar una mayor incidencia en los procesos de regulación de las actuaciones en el espacio urbano.

De acuerdo a los resultados obtenidos para el caso del AMB, se puede aventurar que en dicha metrópolis existe un menor nivel de desigualdad intermunicipal que el que se advierte para el caso de las áreas metropolitanas de América Latina. Desde ya, variados estudios sobre la configuración socioespacial del Área Metropolitana de Santiago (De Mattos C., Riffo, L., Yáñez, G. y Salas, X., 2005; De Mattos, 2008; Orellana y Fuentes, 2007; Orellana, 2007b; Fuentes L., Allard P. y Orellana, A., 2008) permiten afirmar que los municipios del denominado cono

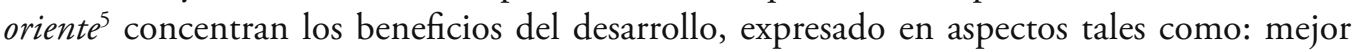
equipamiento urbano de excelencia, mayor disposición de áreas verdes y una provisión de servicios propio de una "ciudad global". Así, los territorios municipales dejan de manifiesto el mayor nivel de empoderamiento económico y político de sus residentes en el contexto metropolitano del AMS, cumpliéndose la máxima que plantea Veltz (1999) de que las metrópolis tienden a parecerse a escala global y a fragmentarse a pequeña escala.

Los municipios mejor posicionados para internalizar los beneficios de la globalización, constituirán la elite de territorios comunales donde se concentrarán estándares de calidad de vida homologables a cualquier ciudad global europea o de Estados Unidos, mientras que el resto de los municipios sólo serán receptores de lo que no es parte del interés de los municipios ganadores, esperando entonces sólo que los posibles beneficios provengan de las actuaciones

\footnotetext{
3 Tesis doctoral del autor, ver bibliografía.

4 Hace referencia al territorio que está bajo jurisdicción municipal, donde reside una población que concentra mayor nivel educacional, de ocupaciones directivas y nivel de ingresos, comparativamente con los residentes del resto de municipios del $\mathrm{AMB}$.

${ }^{5}$ Conformado por los municipios de Santiago, Providencia, Las Condes, Vitacura y Lo Barnechea.
} 
derivadas de la inversión pública, muy probablemente decididas desde el nivel central y que sólo vienen a reafirmar el patrón de desigualdad preexistente (Orellana y Fuentes, 2008). Por tal motivo, la competencia interurbana se alimentará por la imposibilidad de generar respuestas por parte del gobierno central a las necesidades locales, el crecimiento y la complejidad de la vida social, lo que genera a su vez polarización y exclusión, acontecimientos que proyectarán una imagen de aparente caos e ingobernabilidad de la vida en las ciudades (Kearns y Paddison, 2002).

La configuración socioespacial resultante para el AMS, expresada en la geometría que dibuja un cono, un arco y variados cluster en el plano metropolitano, pone de manifiesto aspectos políticos y sociales importantes, ya que da cuenta de un desequilibrio en el proceso de acumulación de poder entre municipios, profundizado por la ausencia de un gobierno metropolitano o una institucionalidad que regule con eficacia y autonomía suficiente los excesos de competitividad intermunicipales, básicamente inducidos por la acción del mercado y las políticas centralizadas que operan, por lo general, sin una visión plenamente metropolitana (Orellana, 2007a).

\section{Metodología de perfiles socioterritoriales y ranking de los municipios del AMS}

Basado en el trabajo de Sánchez (1997) para los municipios de Cataluña, se plantea una metodología para la construcción de los perfiles socioterritoriales de los municipios del AMS. La idea básica aplicada es la de considerar el peso de la población total del municipio dentro del total del AMS, como punto base de referencia que permita posteriormente la comparación intermunicipal entre factores, ocupando la siguiente fórmula para el cálculo del coeficiente:

$$
\text { Coeficiente }=(\% \text { factor }-\% \text { población }) /(\% \text { factor }+\% \text { población })
$$

De esta manera, si el resultado es “ 0 ” (o cercano a cero) se puede señalar que respecto al factor señalado el municipio presenta una proporcionalidad perfecta con el AMS. Si el valor tiende a 1 significa que el municipio presenta dominancia en relación al conjunto del AMS, mientras que si tiende al valor -1 señala una menor presencia de tal factor respecto al conjunto del AMS.

Respecto a la construcción del ranking, sobre la base de los coeficientes obtenidos para los cuatro indicadores que alcanzaron mejores resultados desde el punto de vista de sus coeficientes de correlación, se construyó un ranking que es similar al que se derivó del estudio sobre el Área Metropolitana de Barcelona (Orellana, 2006), donde se realizó un proceso de iteración que permitió ranquear a partir de una variable y luego la otra, así sucesivamente. Cabe destacar que los resultados bajo este método no difieren considerablemente, en cuanto a la ubicación de los municipios, de cuando se utiliza el criterio de igual proporcionalidad que sostienen indicadores como el Índice de Desarrollo Humano (IDH).

\section{Gobierno municipal y perfiles socioterritoriales del AMS}

Por Ley los municipios en Chile están definidos como "Corporaciones autónomas de derecho público, con personalidad jurídica y patrimonio propio, cuya finalidad es satisfacer las necesidades 
de la comunidad local y asegurar su participación en el progreso económico, social y cultural de las respectivas comunas" ", para lo cual tienen como responsabilidades la administración del espacio público, el equipamiento comunal, el tránsito, los servicios de aseo y ornato, y la regulación del área urbana. Por lo tanto, se puede reconocer que en el marco de sus actuaciones el municipio tiene la "capacidad de gobernar, ejercer autoridad y realizar acciones que ordenen, dispongan y organicen el territorio contenido en su jurisdicción con el fin de satisfacer las demandas de su población" en términos de espacio público, equipamiento, tránsito, aseo y ornato y regulación de las áreas urbanas (Fuentes, Allard y Orellana, 2007).

Sin embargo, en la práctica los municipios son instituciones generalmente débiles, con poco poder económico, político e ideológico, limitados en su autonomía, autoridad, legitimidad y capacidad de gestión, con poca claridad en su rol en la vida política local de la ciudad (Rodríguez y Winchester, 1997). Es precisamente en el contexto del AMS donde se hace presente no sólo la debilidad manifiesta de gran parte de los municipios, sino también la condición de privilegio que sustentan unos pocos para abordar con mayor satisfacción las demandas de su población residente (Orellana, 2007b). Se refiere, particularmente, a la red de contactos y articulación social que se establece a partir de la calidad de la educación obtenida por cada individuo (primaria, secundaria y profesional), la categoría socio-ocupacional que sostienen (directivos, medios, empleado-obrero, no calificado $)^{7}$, el perfil socioterritorial comunal ${ }^{8}$ y el predominio de uno u otro sector político en el gobierno municipal ${ }^{9}$. Factores que, en conjunto, definen el perfil socioterritorial de los municipios.

La disparidad manifiesta entre municipios se puede visualizar en la gráfica que se presenta en el Anexo 1, para cuatro comunas del AMS: Cerro Navia, La Pintana, Providencia y Vitacura. Los perfiles de estos municipios representan la expresión más elocuente de las disparidades que existen en términos socioterritoriales. Así, por ejemplo, la población residente de las comunas de Cerro Navia y La Pintana que recurrentemente lideran el ranking del AMS con menor perfil socioeconómico, en términos del perfil educacional de su población potencialmente

\footnotetext{
${ }^{6}$ Ley Orgánica Constitucional de Municipalidades, Ministerio del Interior, 2002.

7 Se entenderá como Directivos aquellos individuos que dentro de las categorías laborales ocupan cargos directivos empresariales y públicos, recibiendo en su totalidad un ingreso que se ubica en el primer quintil de ingresos. Por su parte, se agrupan en Medios individuos que son empresarios o profesionales independientes (no directivos) que reciben ingresos, por lo general, situados en el primer y segundo quintil de ingresos. Luego está la categoría denominada Empleados/Obreros conformado por individuos que son parte de la fuerza de trabajo técnica calificada en el área de la industria, agricultura, turismo y servicios (incluye empleados del sector financiero y administrativos públicos y privados, en general), los cuales suelen tener un ingreso que fluctúa entre el tercer y cuarto quintil. Finalmente, están los individuos No calificados los cuales se desenvuelven en todas las áreas de la actividad productiva desempeńando trabajos, por lo general, de carácter menor, a plazo fijo y con una alta movilidad laboral, recibiendo en su totalidad un ingreso equivalente al último quintil de ingresos, en gran proporción la población en situación de pobreza.

${ }^{8}$ Asumiendo que el territorio municipal se configura con una población que tiene una proporción de población en situación de pobreza, donde la existencia de una alta oferta de matrículas de colegios pagados (no subvencionados) determina un patrón de segregación importante, así como las diferencias relativas en materia de presupuesto e inversión municipal establecen claras señales de las disparidades comunales a escala metropolitana.

${ }^{9}$ En el caso de Chile, se pueden distinguir dos referentes políticos importantes: Alianza por Chile y la Concertación de Partidos Políticos por la Democracia, este último sector gobernante desde el regreso a la democracia en 1990.
} 
activa $^{10}$, muestran severos déficit de profesionales y técnicos comparativamente con el nivel de especialización que alcanza el AMS, contrariamente a lo que ocurre con su población con estudios primarios y secundarios que está por sobre el promedio. En un sentido inverso, comunas como Providencia y Vitacura, acostumbradas a estar en lo alto de los rankings comunales, en materia educacional su población residente muestra un amplio superávit de profesionales y levemente superior de técnicos respecto al AMS.

En cuanto a la comparación del perfil socio-ocupacional entre estas mismas comunas, se constata que las primeras sostienen déficit significativo de población que ocupa cargos directivos y medios en el mercado del trabajo, lo opuesto a lo que ocurre con el segundo par de comunas. Consecuente con lo anterior, el superávit de población que oficia como empleado/obrero y otros no calificados para los primeros se contrapone con los déficits que se expresan para el segundo par de comunas.

Ahora, tomando como referencia algunas variables que permitan aproximarse a la caracterización de las comunas, como son: población en situación de pobreza, inversión total municipal y matrículas de enseñanza particular pagada, se advierte que para las comunas del perfil de Cerro Navia y La Pintana las tres variables muestran resultados deficitarios en términos de especialización respecto al AMS. Por el contrario, las comunas de Providencia y Vitacura reportan amplios superávit para las mismas tres variables consideradas. Y, finalmente, en lo que respecta a la representatividad de los bloques políticos, los resultados no permiten aventurar juicios sobre una incidencia, dado que al correlacionarlos con algunas variables que permitieron construir el ranking de comunas en base al perfil socioterritorial, no se expresó de mucha significancia estadística, arrojando menos del 50\%.

\section{Ranking de los municipios del AMS}

La dirección estatal de la vida de las ciudades ha sido sustituida por las gobernanzas urbanas, que hoy en día se plantean de manera distinta a como antes se acostumbraba. Hoy las gobernanzas ponen énfasis en obtener respuestas prontas y eficaces en un contexto complejo de circunstancias cambiantes (Kearns y Paddison, 2002). Así, podemos arribar a la tesis de que la gobernabilidad territorial está determinada por tres factores claves: autonomía territorial de las comunas, las capacidades endógenas y la disponibilidad de recursos, todo lo cual determina la plataforma de poder para enfrentar los desafíos que se imponen a las autoridades municipales y a los propios agentes de la comunidad local (Fuentes, Allard y Orellana, 2007).

En el informe de Desarrollo Humano para Chile del PNUD (2004), frente a la pregunta sobre cuáles son las instituciones que tienen más poder en Chile, la mayoría de los encuestados manifestó que era el Gobierno, seguido de los empresarios, los partidos políticos y los jueces; mientras que los municipios alcanzan un bajísimo porcentaje de mención, al igual que la gran mayoría de las organizaciones y entidades propias de la sociedad civil: colegios profesionales,

\footnotetext{
${ }^{10}$ Cabe consignar que los perfiles socioterritoriales hacen referencia a la población de 15 años y más, es decir, potencialmente activa para constituir la fuerza de trabajo.
} 
sindicatos, universidades, iglesia evangélica y organizaciones sociales. La percepción de los encuestados coincide con el hecho de que es en dichas esferas donde la categoría dirigencial alcanza su máxima representación.

A partir de los resultados obtenidos por el PNUD (2004), utilizando datos del CENSO (2002) y de la Encuesta CASEN (2003), podemos sostener como hipótesis que una mayor concentración relativa de clase dirigencial en una comuna debiera ir acompañado de una mayor concentración de población con educación profesional y una menor concentración de población en situación de pobreza. Consistente con las variables escogidas en la metodología aplicada para el caso del Área Metropolitana de Barcelona (Orellana, 2006), podemos considerar que la construcción de perfiles socioterritoriales para el AMS es factible de realizar al contemplar dentro de la categoría socio-ocupacional a dirigentes, nivel de educación (profesionales) y nivel de ingreso, usando como proxy el porcentaje de la población en situación de pobreza y el presupuesto municipal, siendo este último además una medida importante del diferencial de autonomía municipal.

La Tabla 1 relativa a los coeficientes de correlación entre las variables socioterritoriales para el AMS, permite corroborar el alto nivel de significancia en la correlación que existe entre dichas variables.

Tabla 1

COEFICIENTES DE CORRELACIÓN ENTRE VARIABLES SOCIOTERRITORIALES RELEVANTES DEL AMS

\begin{tabular}{|c|c|c|c|c|c|}
\hline Variables correlacionadas & $\begin{array}{c}\text { Muy } \\
\text { Negativa }\end{array}$ & Negativa & Neutra & Positiva & $\begin{array}{c}\text { Muy } \\
\text { Positiva }\end{array}$ \\
\hline Educación profesional/Dirigentes & & & & & 0,8 \\
\hline Dirigentes/Poblaciónen situación de & & & & & \\
\hline pobreza & $-0,88$ & & & & \\
\hline Dirigentes/Presupuesto municipal & & & & & 0,77 \\
\hline $\begin{array}{l}\text { Educacional profesional/Presupuesto } \\
\text { municipal }\end{array}$ & & & & 0,62 & \\
\hline $\begin{array}{l}\text { Presupuesto municipal/Población en } \\
\text { situación de pobreza }\end{array}$ & -77 & & & & \\
\hline
\end{tabular}

Por un lado, se advierte la alta correlación negativa que existe entre la población en situación de pobreza respecto a la categoría socio-ocupacional de dirigentes, hecho que implica que en aquellas comunas donde existe una mayor concentración relativa de población en situación de pobreza es donde también hay una menor concentración de directivos públicos y privados y, al mismo tiempo, donde comparativamente los municipios tienen un menor presupuesto municipal para hacer frente a las demandas socioterritoriales de su población residente. Y, por otro lado, existe una alta correlación positiva entre la variable socio-ocupacional directivos y la variable educacional profesionales, lo que en esencia implica que un porcentaje importante 
de aquellos que detentan poder ${ }^{11}$ son profesionales. En coherencia con lo anterior, también se constata que los municipios que sostienen un mayor presupuesto municipal tendrán una mayor concentración de directivos y profesionales como residentes.

Al igual que en el estudio para el Área Metropolitana de Barcelona, para establecer rangos de perfiles socioterritoriales de los municipios del AMS, se consideraron cinco municipios en el nivel superior e igual número en el nivel inferior del ranking. Luego se contempló un criterio de igual proporcionalidad de municipios para el caso de los municipios ubicados en el ranking en un nivel bajo (pero no extremo) respecto a la media del AMS; un nivel medio para aquellos cuyo perfil socioterritorial es similar al promedio de la metrópolis; $y$, por último, un nivel alto para los que están sobre ese promedio pero no alcanzan el nivel superior.

Tomando en consideración las variables socioterritoriales referidas, fue posible construir un ranking de municipios del AMS en base al método planteado por Sánchez (1997). Este ejercicio intentó aportar evidencia sobre el nivel de empoderamiento relativo de los municipios del AMS, considerando cuánto sobre o bajo un nivel promedio metropolitano (proporcional a la población) se encontraba cada uno de los 34 municipios. El resultado se expresa en la Tabla 2 , donde queda de manifiesto que es precisamente en los municipios del denominado cono oriente (Providencia, Vitacura, Las Condes, Lo Barnechea y Santiago) donde se concentra el perfil socioterritorial con mayor poder relativo dentro del AMS.

Utilizando las categorías de análisis aplicadas para el caso del AMB (Orellana, 2006), los cinco municipios que conforman el cono oriente se encuentran en el extremo superior del ranking (ver Mapa 1). Mientras que el extremo inferior, lo conforman los municipios de La Pintana, Cerro Navia, Lo Espejo, San Ramón y Renca. Así, por ejemplo, mientras que en las comunas del extremo superior la concentración de directivos que residen se mueve entre un rango de $33 \%$ y $61 \%$ por sobre el promedio metropolitano, en las comunas del extremo inferior están entre un rango entre $66 \%$ y un $77 \%$ por debajo del promedio, es decir, son altamente deficitarios en términos de población que ocupe puestos directivos en el mercado del trabajo. Tal tendencia, en menor o mayor medida, queda reflejada para todas las variables socioterritoriales sobre las cuales se construyó el ranking en cuestión, es decir, para la concentración de profesionales, población en situación de pobreza y presupuesto municipal.

Otro aspecto interesante de rescatar de la gráfica que arroja el Mapa 1, tiene que ver con el proceso de degradación en el perfil socioterritorial que produce alejarse en sentido suroeste, donde prima un perfil socioterritorial bajo, sin perjuicio que son las comunas de La Pintana, Lo Espejo, San Ramón, Cerro Navia y Renca las que marcan el extremo opuesto del ranking de las comunas del llamado cono oriente de Santiago. Del mismo modo, cabe hacer notar que las comunas que muestran un perfil socioterritorial alto (Huechuraba, La Reina, Nuñoa, Peñalolén) se encuentran mayoritariamente colindantes a las comunas del extremo superior del ranking.

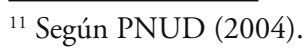


Tabla 2

RANKING DE MUNICIPIOS DEL AMS SEGÚN COEFICIENTES DE ESPECIALIZACIÓN

\begin{tabular}{|c|c|c|c|c|c|c|c|}
\hline Lugar & Municipio & $\begin{array}{l}\text { Superficie } \\
\text { (hás.) }\end{array}$ & Profesional & Dirigentes & $\begin{array}{c}\text { Población en } \\
\text { Situación de } \\
\text { Pobreza }\end{array}$ & $\begin{array}{c}\text { Presupuesto } \\
\text { municipal }\end{array}$ & Población \\
\hline 1 & Providencia & $-0,07$ & 0,51 & 0,61 & $-0,80$ & 0,61 & 120.874 \\
\hline 2 & Vitacura & 0,35 & 0,14 & 0,61 & $-0,91$ & 0,51 & 81.499 \\
\hline 3 & Las Condes & 0,12 & 0,14 & 0,59 & $-0,97$ & 0,13 & 249.843 \\
\hline 4 & Lo Barnechea & 0,60 & 0,23 & 0,37 & $-0,13$ & 0,31 & 74.749 \\
\hline 5 & Santiago & $-0,09$ & 0,27 & 0,33 & $-0,24$ & 0,58 & 200.792 \\
\hline 6 & San Miguel & $-0,06$ & 0,09 & 0,20 & $-0,29$ & 0,06 & 78.872 \\
\hline 7 & Ñuñoa & $-0,11$ & 0,37 & 0,14 & $-0,71$ & 0,17 & 163.511 \\
\hline 8 & La Reina & 0,19 & 0,31 & 0,14 & $-0,58$ & 0,06 & 96.762 \\
\hline 9 & Macul & $-0,09$ & 0,01 & 0,01 & 0,03 & $-0,10$ & 112.535 \\
\hline 10 & La Florida & $-0,08$ & $-0,06$ & $-0,02$ & $-0,18$ & $-0,27$ & 365.674 \\
\hline 11 & La Cisterna & $-0,08$ & $-0,07$ & $-0,07$ & $-0,03$ & $-0,27$ & 85.118 \\
\hline 12 & Peñalolén & 0,05 & $-0,19$ & $-0,08$ & 0,03 & $-0,36$ & 216.060 \\
\hline 13 & Huechuraba & 0,33 & $-0,24$ & $-0,10$ & 0,13 & 0,01 & 74.070 \\
\hline 14 & Independencia & $-0,10$ & $-0,11$ & $-0,12$ & $-0,12$ & $-0,11$ & 65.479 \\
\hline 15 & Quinta Normal & $-0,09$ & $-0,31$ & $-0,13$ & 0,12 & $-0,18$ & 104.012 \\
\hline 16 & Recoleta & $-0,13$ & $-0,36$ & $-0,13$ & 0,08 & $-0,18$ & 148.220 \\
\hline 17 & Puente Alto & 0,03 & $-0,13$ & $-0,13$ & 0,03 & $-0,39$ & 492.915 \\
\hline 18 & Conchalí & $-0,14$ & $-0,13$ & $-0,14$ & 0,17 & $-0,19$ & 133.256 \\
\hline 19 & Estación Central & $-0,11$ & $-0,26$ & $-0,21$ & $-0,05$ & $-0,17$ & 130.394 \\
\hline 20 & Maipú & $-0,08$ & $-0,24$ & $-0,23$ & $-0,13$ & $-0,21$ & 468.390 \\
\hline 21 & San Joaquín & $-0,11$ & $-0,33$ & $-0,37$ & 0,07 & $-0,11$ & 97.675 \\
\hline 22 & San Bernardo & 0,17 & $-0,13$ & $-0,39$ & 0,16 & $-0,13$ & 246.762 \\
\hline 23 & Quilicura & 0,31 & $-0,13$ & $-0,40$ & $-0,31$ & 0,03 & 126.518 \\
\hline 24 & Lo Prado & $-0,37$ & $-0,34$ & $-0,45$ & 0,06 & $-0,33$ & 104.316 \\
\hline 25 & El Bosque & $-0,26$ & $-0,14$ & $-0,50$ & 0,24 & $-0,32$ & 175.594 \\
\hline 26 & $\begin{array}{l}\text { Pedro Aguirre } \\
\text { Cerda }\end{array}$ & $-0,29$ & $-0,13$ & $-0,52$ & 0,04 & $-0,22$ & 114.560 \\
\hline 27 & Pudahuel & 0,03 & $-0,51$ & $-0,53$ & 0,05 & $-0,31$ & 195.653 \\
\hline 28 & Cerrillos & 0,26 & $-0,55$ & $-0,58$ & $-0,17$ & 0,12 & 71.906 \\
\hline 29 & La Granja & $-0,30$ & $-0,77$ & $-0,62$ & 0,26 & $-0,20$ & 135.520 \\
\hline 30 & Renca & 0,12 & $-0,63$ & $-0,66$ & 0,31 & $-0,22$ & 133.518 \\
\hline 31 & San Ramón & $-0,31$ & $-0,60$ & $-0,66$ & 0,36 & $-0,20$ & 94.906 \\
\hline 32 & Lo Espejo & $-0,31$ & $-0,68$ & $-0,68$ & 0,31 & $-0,26$ & 112.800 \\
\hline 33 & Cerro Navia & $-0,29$ & $-0,69$ & $-0,69$ & 0,30 & $-0,17$ & 148.312 \\
\hline 34 & La Pintana & $-0,06$ & $-0,77$ & $-0,77$ & 0,14 & $-0,23$ & 190.085 \\
\hline
\end{tabular}




\section{Mapa 1 \\ PERFIL SOCIOTERRITORIAL DE LAS COMUNAS DEL AMS ${ }^{13}$}

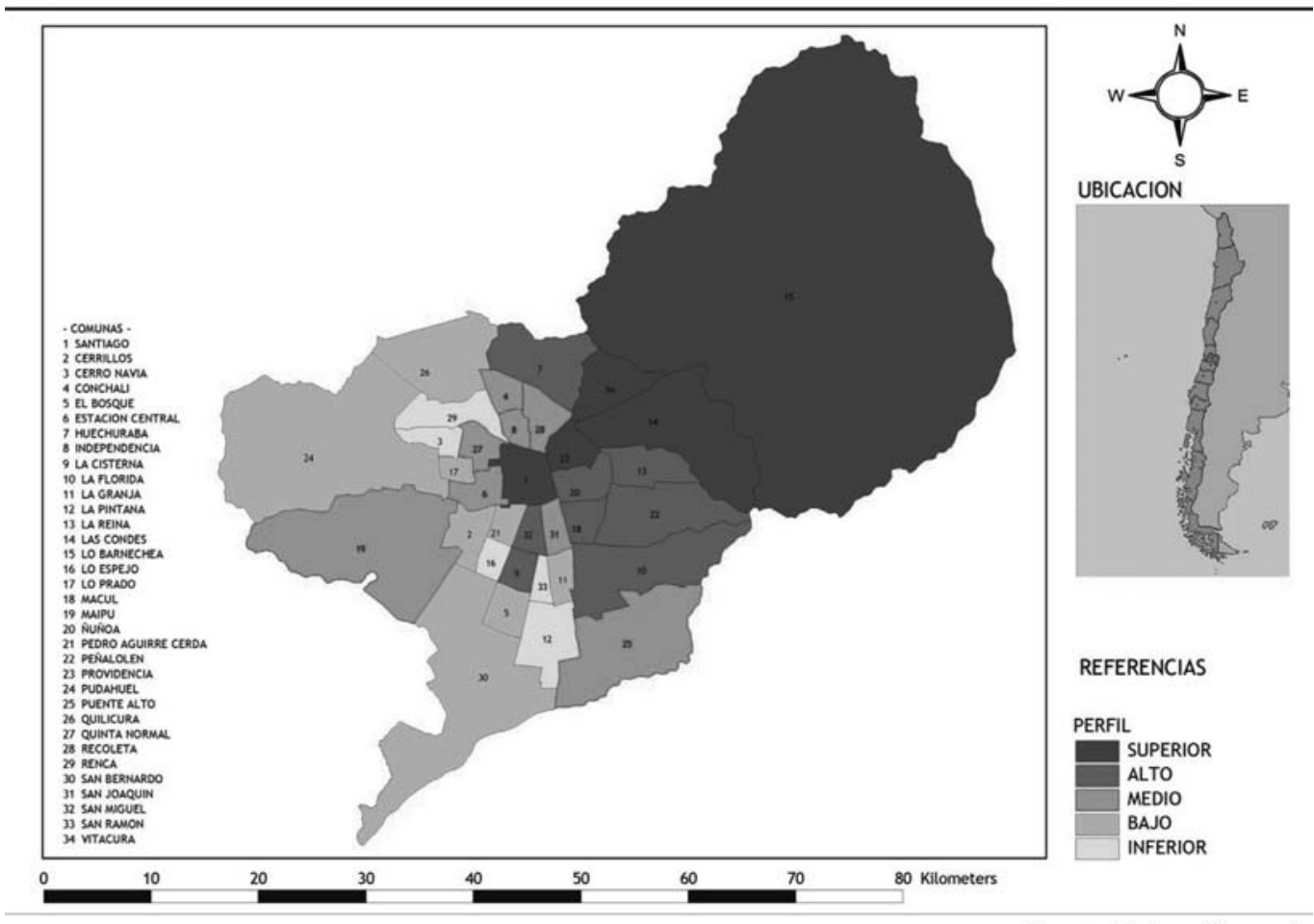

Fuente: Elaboración propia

Para reforzar la validez del análisis comparativo que muestra el gran diferencial entre los municipios del extremo superior e inferior del ranking resultante, vale la pena detenerse en otras variables incorporadas al ranking de la Tabla 2. Al considerar la superficie urbana disponible y potencial, al interior de cada grupo extremo de comunas, se advierte una situación de disparidad, donde se presentan municipios tanto con déficit como con superávit relativo, de cuyo comportamiento se deduce que no es posible obtener una correlación significativa de esta variable asociada a la condición de mayor o menor perfil socioterritorial obtenida en el ranking. Así también, comparando la población de cada comuna, podemos también deducir que esta variable no es explicativa de las marcadas diferencias entre cada extremo, ya que la proporcionalidad y la suma de población entre cada grupo no es significativamente distinta.

No obstante, resulta muy revelador de la gran disparidad entre los perfiles socioterritoriales de los municipios del extremo superior con los del extremo inferior, cuando se compara el hecho que los primeros concentran el $42 \%$ del presupuesto de todos los municipios del AMS ${ }^{12}$, mientras que los segundos sólo alcanzan el $8 \%$ de dicho presupuesto, considerando que concentran una población más o menos equivalente, 13\% y 11,6\%, respectivamente. Más aún,

\footnotetext{
${ }^{12} \mathrm{Al} 2006$ el presupuesto alcanzaba un equivalente a 180 millones de dólares, según datos SINIM (SUBDERE, 2006).
} 
si se toma en consideración el resultado obtenido en Orellana (2007b), donde se advertía que eran los mismos municipios del extremo superior quienes detentaban una dependencia del $\mathrm{FCM}^{13}$ inferior al $10 \%$ en contraposición a los del extremo inferior, donde la dependencia del FCM promediaba el 50\%. Todo lo anterior, permite concluir que no sólo los municipios del extremo superior alcanzan una condición de mayor poder relativo derivado de su perfil socioterritorial y disponibilidad de recursos, sino también de mayor autonomía en su gestión al no depender en forma importante del FCM, señal a la vez inequívoca de la capacidad de generar recursos propios de los municipios.

\section{Reflexiones finales}

En un trabajo reciente publicado por De Mattos $(2008)^{14}$ sobre los cambios socio-ocupacionales y la transformación metropolitana de Santiago entre 1992-2002, se ratifica que la alta concentración de directivos y profesionales que mostraban en el año 1992 las cinco comunas que conforman el cono oriente del AMS (y que en este estudio representan el extremo superior del ranking de perfiles socioterritoriales), para el año 2002 elevan considerablemente su concentración, si bien se empieza a expandir la concentración hacia comunas colindantes, como Huechuraba y Peñalolén. Consistente con este fenómeno, un importante periódico de cobertura nacional ${ }^{15}$ publicaba un sondeo que decía que el 50\% de los gerentes de grandes empresas habían egresado de cinco colegios (Verbo Divino, Colegio de los Sagrados Corazones de Manquehue, Saint George, San Ignacio y Tabancura), todos particulares vinculados a la Iglesia Católica y localizados en comunas de nuestro extremo superior del ranking. Se agregaba, además, como resultado de este sondeo, que el 84\% de los gerentes de las grandes empresas había estudiado en colegios particulares, es decir, sin ningún subsidio del Estado.

Por otro lado, Moris (2008) señalaba acertadamente, como parte de las conclusiones de su trabajo para promover la regeneración urbana, la necesidad de que "los municipios planifiquen el territorio comunal estableciendo zonas especiales o zonas de gestión integrada que enfrenten procesos de deterioro con planes de gestión ad-hoc y que puedan utilizar subsidios más flexibles. Esto significa, según el autor, desarrollar planes de gestión integrada para distritos y barrios; desarrollar normativas específicas de diseño urbano, guías de diseño, inversiones urbanas (vialidades, espacios públicos, áreas verdes); e incorporar a las comunidades y al sector privado en la definición de planes. Sin embargo, las posibilidades de enfrentar el desafío planteado se contrastan con la complejidad que revisten las importantes disparidades que ya se advierten en De Mattos (2008) y De Mattos, Riffo, Yáñez y Salazar (2005), pero sobre todo a partir de los resultados obtenidos en esta investigación.

La búsqueda de un mejor posicionamiento de los municipios en un escenario metropolitano donde más de dos tercios de la inversión pública efectiva (Orellana, 2007a) se decide centralizadamente, y cuando la máxima autoridad del Gobierno Regional, el Intendente -de acuerdo al Artículo 1 de la Ley Orgánica Constitucional sobre Gobiernos y Administración

\footnotetext{
${ }^{13}$ Fondo Común Municipal.

14 A partir de los resultados de una investigación FONDECYT, ver bibliografía.

15 Diario La Tercera, noticia publicada el 30 de marzo de 2008.
} 
Regional- es el representante del Presidente de la República en la región y no el representante de la región ante el Presidente de la República, como suele creerse a veces, hace que los municipios se vean obligados a competir en condiciones de desigualdad en términos de sus activos socioterritoriales, en gran medida resultado de decisiones mayoritariamente exógenas a los intereses de los propios agentes locales, como son: localización de viviendas sociales, vertederos y cárceles, trazados de autopistas urbanas, entre otros. En tal sentido, se cumple lo que señala Müller (2004) para la ciudad de Santiago, la cual se caracteriza por tener un alto grado de competencia entre comunas y una fragmentación creciente de la estructura territorial, con comunas más y menos avanzadas en los procesos de desarrollo.

La globalización, al asociar sociedades locales diversas en un mismo proceso productivo, añade a la diferenciación social una diferenciación territorial (Ascher, 2004). De alguna forma, las actuaciones derivadas desde el ámbito de las políticas públicas y el sector privado, han ido configurando una morfología urbana con marcados contrastes que se manifiestan a escala comunal, agravados por cierta tendencia a la concentración de factores asociados a un mayor poder socioterritorial que impactan a escala metropolitana. Aspectos relativos a las capacidades endógenas, autonomía territorial y disponibilidad de recursos determinan en la práctica las mayores o menores posibilidades de que un municipio resuelva las demandas de su población residente y al mismo tiempo inserte a su comuna con éxito en la dinámica del desarrollo de los espacios globales.

En definitiva, bajo un proceso acelerado de organización política y económica a escala mundial que está articulándose sobre la base de una red de grandes metrópolis, que a su vez está estructurada en base a una red jerarquizada de ciudades, la jerarquía urbana también refleja el papel de ciertos municipios respecto a otros del entorno metropolitano o regional. La base de la competitividad de las metrópolis, no puede entonces ni debe sustentarse sobre la base de la exclusión de ciertos municipios de los beneficios de la globalización, a través de la sustentación de una estructura socioespacial desigual a escala municipal.

\section{Referencias bibliográficas}

Ascher, F. (2004). Los nuevos principios del urbanismo. Madrid: Editorial Alianza.

Borja, J. \& Castells, M. (1998). Local y global. Madrid: Editorial Taurus.

Borja, J. (2008). Gobernabilidad y planeamiento de los territorios metropolitanos. En G. Yáńez; A. Orellana; O. Figueroa \& F. Arenas (Eds.), Ciudad, poder, gobernanza (pp. 135-174). Colección RIDEAL/ Serie GEOLibros No9/EURE Libros. Santiago: Editorial LOM.

Capel, H. (2003). Redes, chabolas y rascacielos. Las transformaciones físicas y la planificación en las áreas metropolitanas. En A. Orellana (Coord.), El desafío de las áreas metropolitanas en un mundo globalizado: una mirada a Europa y América Latina (pp. 17-56). Colección RIDEAL. Barcelona: Institut de Estudios Territoriales.

Centellas i Portella, J. (2002). El buen gobierno de la ciudad. Estrategias urbanas y politica relacional. Instituto Nacional de Administración Pública. Madrid: Plural Editores.

De Mattos, C. (2008). Cambios socio-ocupacionales y transformación metropolitana del Gran Santiago, 1992-2002. En P. Allard (Ed.), Mercado y ciudad: desafíos de un país urbano. Observatorio de Ciudades UC/BBVA. Santiago: Editorial Valente. 
De Mattos, C.; Riffo, L.; Yáñez, G. \& Salas, X. (2005). Reestructuración del mercado metropolitano de trabajo y cambios socio-teritoriales en el Gran Santiago. Santiago, Informe de Investigación Proyecto FONDECYT 1040838.

Delgado, M. (1999). El animal público. Colección Argumentos. Barcelona: Editorial Anagrama.

Fuentes, L.; Allard, P. \& Orellana, A. (2007). El municipio y la gobernabilidad del territorio comunal. En T. Matus (Coord.), La reforma municipal en la mira (pp. 43-84). Santiago: Expansiva/Observatorio de Ciudades UC/Escuela de Trabajo Social UC.

Harvey, D. (2000). Espacios de esperanza. Madrid: AKAL Ediciones.

Instituto Nacional de Estadísticas, INE (1992-2002). Censos de Población. Santiago: INE.

Kearns, A. \& Paddison, R. (2000). New challenges for urban governance. Urban Studies, 37 , 5-6, 845-850.

Ministerio de Planificación (2003). Encuesta CASEN. Santiago: MIDEPLAN.

Ministerio del Interior (2005). Ley Orgánica Constitucional sobre Gobiernos y Administración Regional D.F.L. 1-19.175, Chile.

Ministerio del Interior. Ley Orgánica Constitucional de Municipalidades $N^{\circ} 18.695$.

Molotch, H. (1976). The city as a growth machine: toward a political economy of place. The American Journal of Sociology, 82, 2.

Moris, R. (2008). Regeneración urbana: consolidación y sustentabilidad de un mercado en desarrollo. En P. Allard (Ed.), Mercado y ciudad: desafíos de un pais urbano. Observatorio de Ciudades UC/BBVA. Santiago: Editorial Valente.

Müller, B. (2004). Desafíos de la gobernabilidad-competitividad y habitabilidad de la Región Metropolitana de Santiago. Presentación Conferencia/Taller Gobierno Regional y Territorio Metropolitano, GTZ, Santiago de Chile.

Orellana, A. \& Fuentes, L. (2008). El arco, los conos y los clusters: geometrías espaciales para la gobernabilidad metropolitana y local de Santiago. En G. Yáńez; A. Orellana; O. Figueroa \& F. Arenas (Eds.), Ciudad, poder, gobernanza (pp. 111-132). Colección RIDEAL/ Serie GEOLibros No9/EURE Libros. Santiago: Editorial LOM.

Orellana, A. (2005). El control social del espacio metropolitano desde lo local. En C. De Mattos; O. Figueroa; A. Orellana \& G. Yáñez (Eds.), Gobernanza, competitividad y redes: la gestión de las ciudades del siglo XXI (pp. 263-296). Colección Rideal /Eure Libros. Santiago: LOM Editores. 
Orellana, A. (2006). La incidencia de la configuración socioterritorial en la gobernabilidad metropolitana de Barcelona. Tesis Doctoral, Departamento de Geografía Humana, Universitat de Barcelona (no publicada).

Orellana, A. (2007a). La gobernabilidad metropolitana de las periferias de Santiago. Revista Anfora, 14, 23, 185-198.

Orellana, A. (2007b). La gobernabilidad metropolitana: nuevos escenarios para el desarrollo urbano y territorial del área metropolitana de Santiago. En C. De Mattos \& R. Hidalgo (Eds.), Santiago de Chile: movilidad espacial y reconfiguración metropolitana (pp. 189-206). Colección EURE Libros/GEOLibros N8. Santiago: LOM Ediciones.

Programa Naciones Unidas para el Desarrollo (2004). Informe sobre el Desarrollo Humano en Chile. Santiago: PNUD.

Rodríguez, A. \& Winchester, L. (1997). Fuerzas globales, expresiones locales: desafíos para el gobierno de la ciudad en América Latina. En A. Rodríguez \& L. Winchester (Eds.), Ciudades y gobernabilidad en América Latina (pp. 29-62). Santiago: Ediciones SUR.

Rojas, E.; Cuadrado-Roura, J.R. \& Fernández Güell, J.M. (2005). Las áreas metropolitanas frentes al desafío de la competitividad. En E. Rojas; J.R. Cuadrado-Roura \& J.M. Fernández-Güell (Eds.), Gobernar las metrópolis (pp. 63-126). Washington: BID.

Sánchez, J-E. (1991). Espacio, economía y sociedad. Madrid: Editorial Siglo XXI.

Sánchez, J-E. (1997). Perfil Sòcio-econòmic dels municips catalans. Instruments per a la gestió. Barcelona: Fundació CIREM.

Sánchez, J-E. (2005) ¿Qué gobernabilidad? En C. De Mattos; O. Figueroa; A. Orellana \& G. Yáñez (Eds.), Gobernanza, competitividad y redes: la gestión de las ciudades del siglo XXI (pp. 235-262). Colección Rideal /Eure Libros. Santiago: LOM Editores.

Sassen, S. (2003). Globalization or denationalization? Review of International Political Economy, $10,1,1-22$.

Smith, M. (2002). La reconstitución del Estado: el impacto de las redes intra e intergubernamentales. En J. Subirats (Coord.), Redes, territorios y gobierno. Nuevas respuestas locales a los retos de la globalización (pp. 373-390). Barcelona: Edición Diputación de Barcelona.

Soja, E. (2000). Postmetropolis: critical studies of cities and regions. U.S.A: Blackwell Publisher.

Subsecretaría de Desarrollo Regional (2000-2006). Sistema Nacional de Información Municipal. Chile.

Veltz, P. (1999). Mundialización, ciudad y territorios. Madrid: Editorial Ariel. 


\section{Anexos}

Anexo 1

COEFICIENTE DE ESPECIALIZACIÓN RESPECTO AMS

\section{CERRO NAVIA}

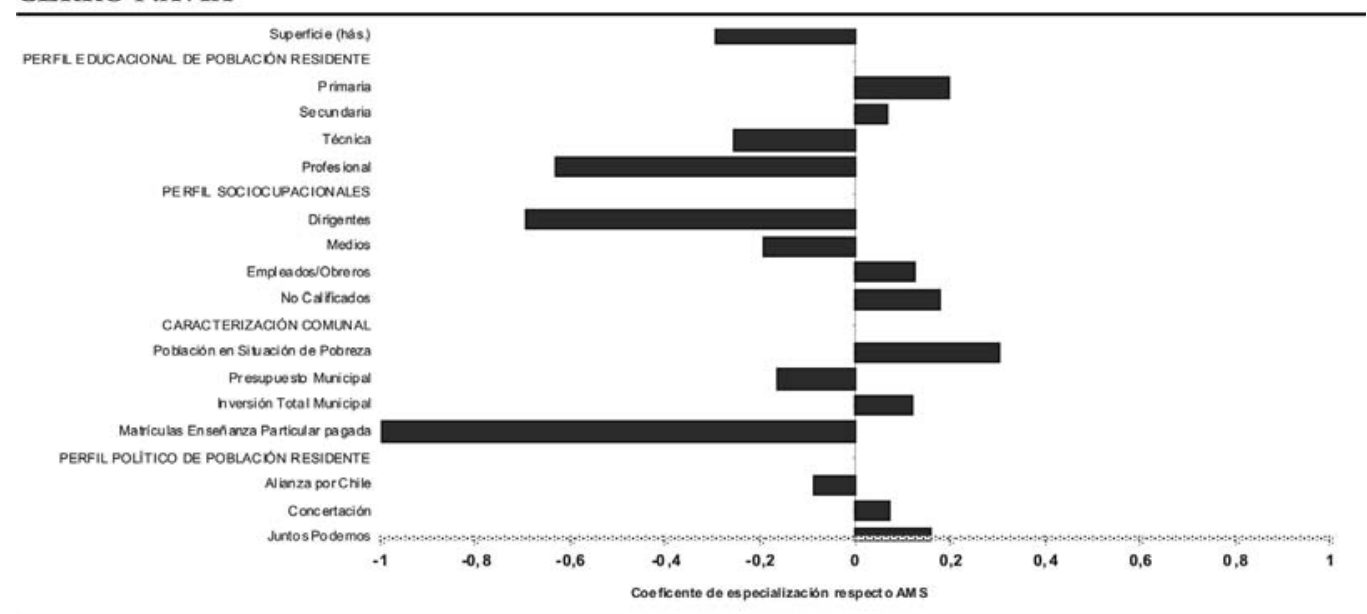

Fuente: Elaboración propia

\section{Anexo 2}

COEFICIENTE DE ESPECIALIZACIÓN RESPECTO AMS

\section{LA PINTANA}

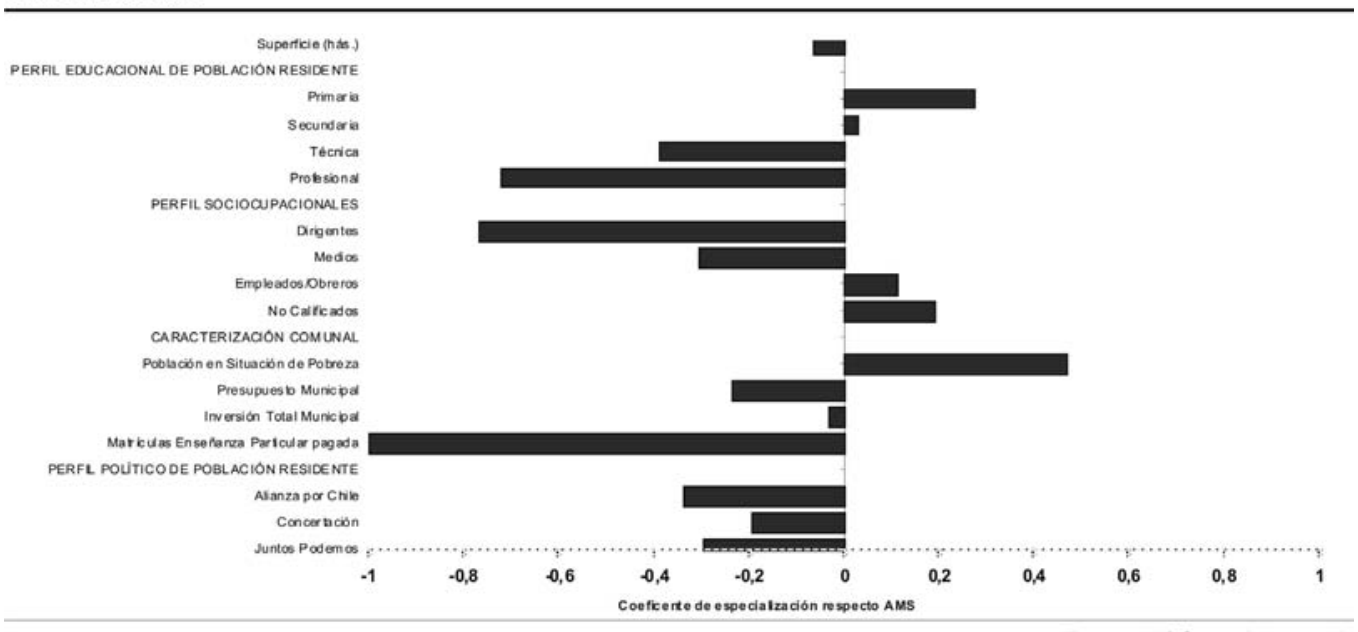

Fuente: Elaboración propia 
Anexo 3

COEFICIENTE DE ESPECIALIZACIÓN RESPECTO AMS

PROVIDENCIA

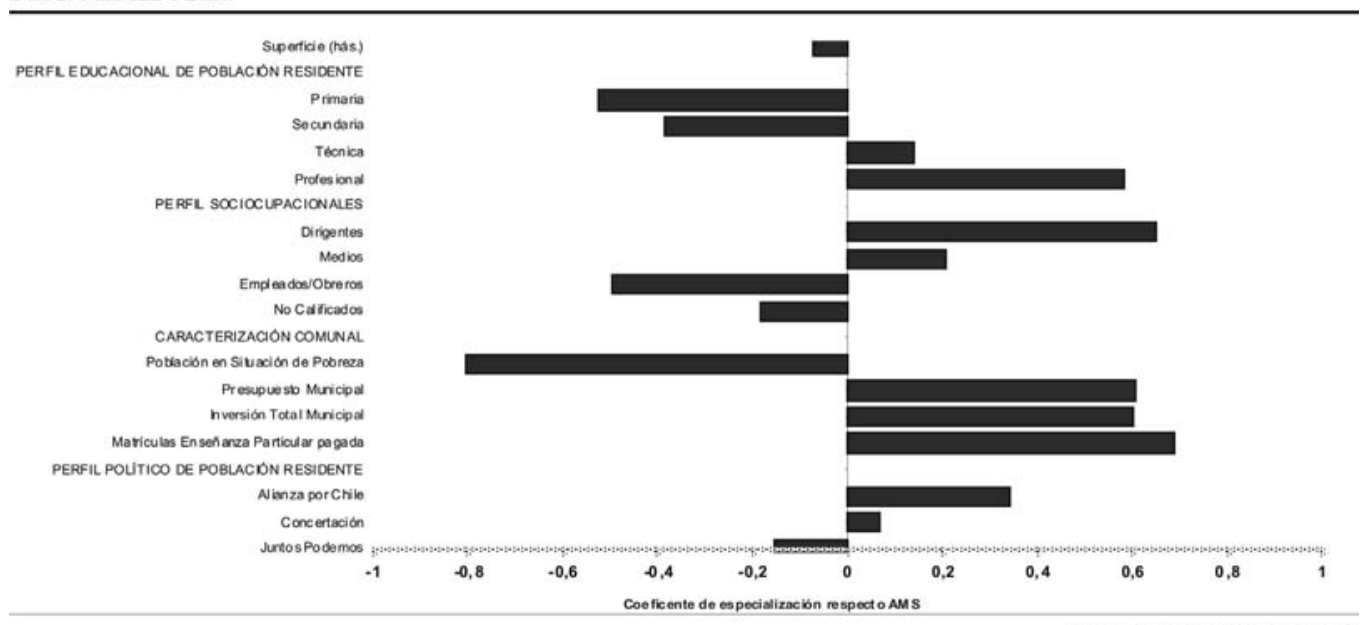

Fuente: Elaboración propia

Anexo 4

COEFICIENTE DE ESPECIALIZACIÓN RESPECTO AMS

VITACURA

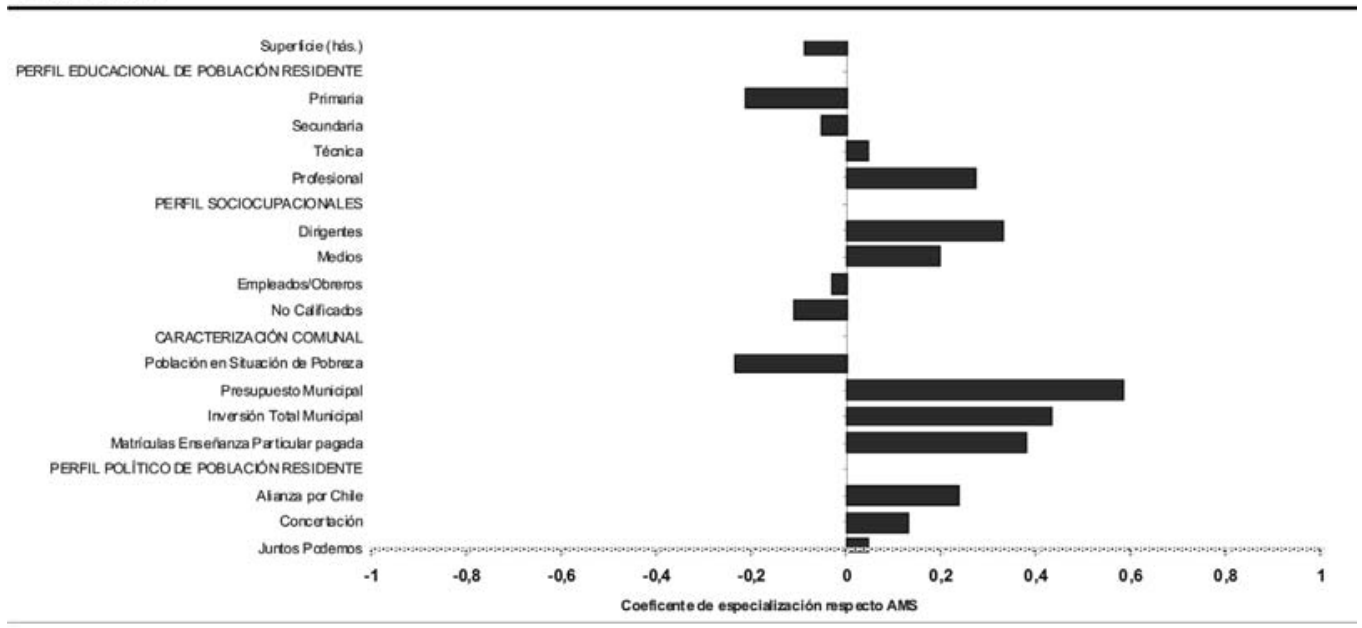

Fuente: Elaboración propia 
